\title{
Particle acceleration in $\eta$ Carinae and cosmic-rays
}

\author{
Roland WALTER* \\ INTEGRAL Science Data Centre, Université de Genève, Chemin d'Ecogia 16, CH-1290 Versoix, \\ Switzerland \\ E-mail: Roland.Walter@unige.ch
}

\section{Christian FARNIER}

INTEGRAL Science Data Centre, Université de Genève, Chemin d'Ecogia 16, CH-1290 Versoix, Switzerland

\section{Jean-Christophe LEYDER}

INTEGRAL Science Data Centre, Université de Genève, Chemin d'Ecogia 16, CH-1290 Versoix, Switzerland

\begin{abstract}
Eta Carinae is the colliding wind binary with the largest mass loss rate in our Galaxy and the only one in which hard X-ray emission has been detected. Eta Carinae is therefore a primary candidate to search for particle acceleration by probing its gamma-ray emission. We used the first 21 months of Fermi/LAT data to extract gamma-ray (0.2-100 GeV) images, spectra and light-curves, then combined them with multi-wavelength observations to model the non-thermal spectral energy distribution. A bright gamma-ray source is detected at the position of eta Carinae. Its flux at a few $100 \mathrm{MeV}$ corresponds very well to the extrapolation of the hard X-ray spectrum towards higher energies. The spectral energy distribution features two distinct components. The first one extends over the $\mathrm{keV}$ to $\mathrm{GeV}$ energy range, and features an exponential cutoff at $\sim 1 \mathrm{GeV}$. It can be understood as inverse Compton scattering of ultraviolet photons by electrons accelerated up to $\gamma \sim 10^{4}$ in the colliding wind region. The second component is a hard gamma-ray tail detected above $20 \mathrm{GeV}$. It could be explained by $\pi^{0}$-decay of accelerated hadrons interacting with the dense stellar wind. The ratio between the fluxes of the $\pi^{0}$ and inverse Compton components is roughly as predicted by simulations of colliding wind binaries. This hard gamma-ray tail can only be understood if emitted close to the wind collision region. The energy transferred to the accelerated hadrons ( $\sim 5 \%$ of the collision mechanical energy) is comparable to that of the thermal X-ray emission.
\end{abstract}

8th INTEGRAL Workshop, The Restless Gamma-ray Universe, Integral2010,

September 27-30, 2010

Dublin Ireland

${ }^{*}$ Speaker. 


\section{Introduction}

The sources of the galactic cosmic rays shall accelerate particles beyond to $10^{15} \mathrm{eV}$ [1] and account for a proton luminosity of $(7-8) \times 10^{40} \mathrm{erg} / \mathrm{s}$ [2], or $10^{50} \mathrm{ergs}$ per Supernova. Cosmicrays have high metallicities and feature abundances characteristic of $\mathrm{OB}$ associations with a mixing of Solar (80\%) and WR ejecta (20\%) [3, 4], suggesting that their acceleration is related to stellar formation and massive stars.

Recent results from Fermi and Cherenkov Telescopes on bright young SNR indicate that their spectra peak at $\mathrm{TeV}$ energies and could be dominated by electron emission [5]. Instead, the spectra of middle aged SNR, interacting with molecular clouds [6, 7], peak at $\mathrm{GeV}$ energies and could be explained by $\pi^{0}$ emission. The particle break energy, derived from their gamma-ray spectra, indicate however a cutoff energy of $10^{10-11} \mathrm{eV}$ much lower than the knee of the cosmic ray spectrum. It is therefore still unclear if SNR can account for the galactic cosmic rays.

About 30 early-type stellar systems feature synchrotron radiation in the radio domain, a signature of electron acceleration [8]. Diffusive shock acceleration in stellar wind collisions [9], either in colliding wind binaries or $\mathrm{OB}$ associations, is the most likely acceleration process and a candidate for cosmic ray acceleration [10, 11]. Gamma-rays, emitted by hadrons accelerated in stellar wind collisions, have however not yet been identified. Located at a distance of $2.3 \mathrm{kpc}$ [12], $\eta$ Carinae is one of the most massive $\left(80-120 M_{\odot}[13,14]\right)$ and brightest stellar system in the Galaxy and features the strongest mass loss rate known. As, in addition, its colliding wind region is relatively wide, $\eta$ Carinae is a primary candidate for gamma-ray detection.

A hard X-ray tail had first been observed towards $\eta$ Carinae by BeppoSAX [15], and has subsequently been unambiguously confirmed by both INTEGRAL [16, 17] and Suzaku [18] observations. This gives a strong evidence that the wind collision leads to non-thermal electron acceleration, and suggests the possibility of a gamma-ray detection.

\section{Fermi/LAT observations}

We have used Fermi/LAT data accumulated in the Carina region during 21 months, from August 4, 2008 to April 3, 2010. The Fermi source FGL J1045.0-5942 with $\alpha=161.265$ and $\delta=-59.7015$ (95\% confidence radius of $1.18 \mathrm{arcmin})$ is perfectly compatible with the position of $\eta$ Carinae. The presence of the source is indubitable, with a test statistic TS $>2800(\approx 53 \sigma)$ for the $200 \mathrm{MeV}$ to $100 \mathrm{GeV}$ energy range. Given the number of pulsars detected within $2^{\circ}$ of the Galactic plane the probability to find one of them in the $95 \%$ confidence region of $\eta$ Carinae is $2.5 \times 10^{-5}$. Moreover, we conducted a blind search frequency analysis and did not find any evidence for a pulsation in FGL J1045.0-5942.

The spectral analysis of FGL J1045.0-5942 was performed using the maximum likelihood method. The region modeling includes two diffuse emissions (Galactic plane and isotropic) and 35 point-like sources listed in the 1FGL catalogue. The resulting spectral energy distribution are presented in Fig. 1. A curvature at low energy and a modification of the spectral slope at highenergy are clearly detected. 


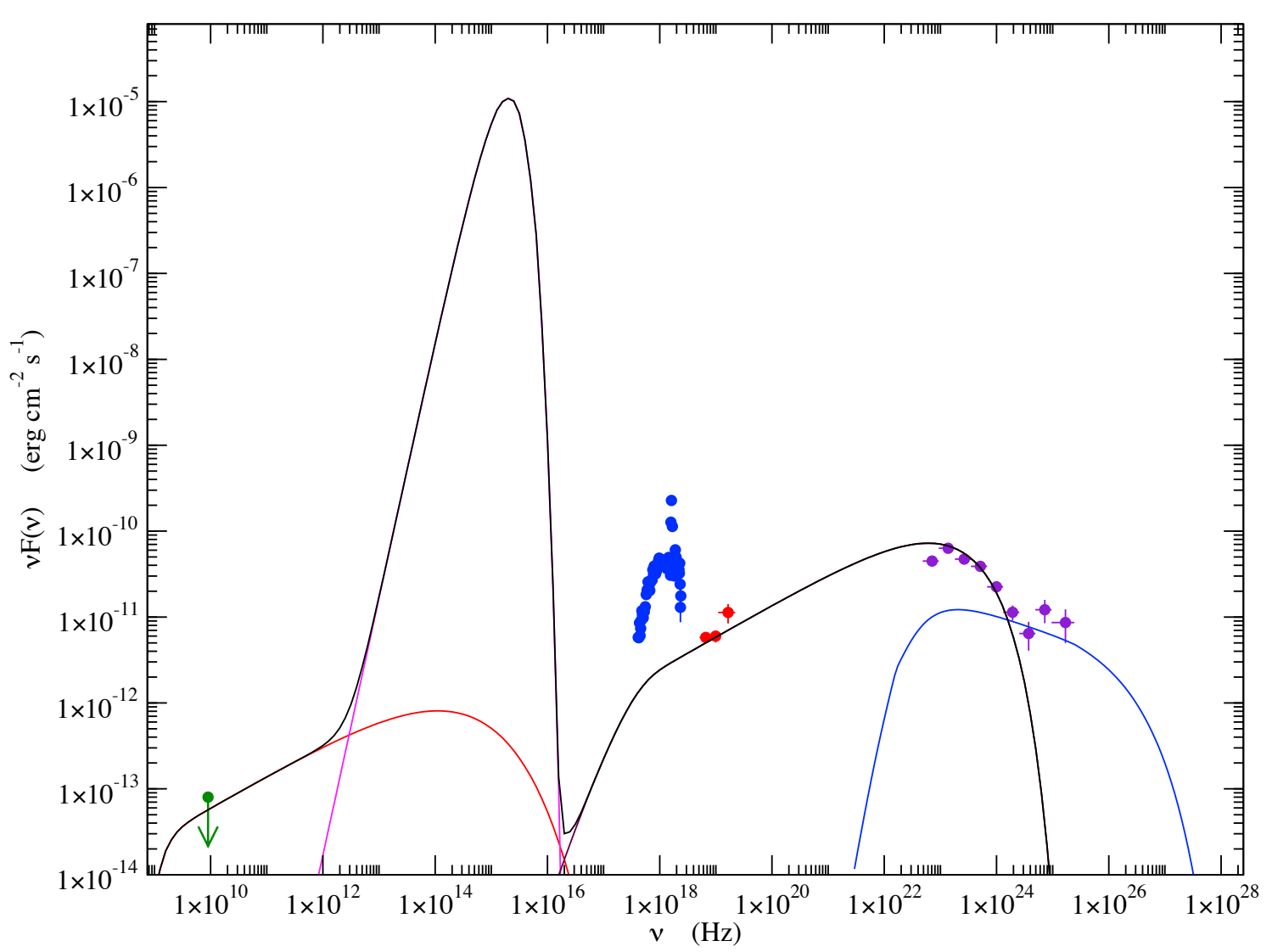

Figure 1: Spectral energy distribution of $\eta$ Carinae including BeppoSAX/MECS (blue), INTEGRAL/ISGRI (red) and Fermi/LAT (purple) data, and a radio upper limit to the synchrotron emission (green). From low to high energies are shown the synchrotron, stellar emission, inverse Compton and $\pi^{0}$-decay spectral components.

\section{Particle acceleration in $\eta$ Carinae}

In the shock region, particle acceleration is counterbalanced by two main cooling processes. (A) Inverse Compton scattering of electrons in the intense ultraviolet radiation field of the stars. The cooling time scale is

$$
t_{I C}=\frac{3 \gamma m_{e} c^{2}}{4 \sigma_{T} c \gamma^{2} \beta^{2} U_{r a d}}=\frac{3 \pi R^{2} m_{e} c^{2}}{\sigma_{T} \gamma \beta^{2} L} \approx \frac{R_{10^{14} \mathrm{~cm}}^{2}}{\gamma_{10^{4}} L_{5 \cdot 10^{6} \mathrm{~L}_{\odot}}} \times 6 \cdot 10^{2} \mathrm{~s}
$$

where $L$ is the stellar ultraviolet luminosity of the primary star and $R$ is its distance to the colliding wind region. (B) Proton-proton interactions and subsequent pion decay. The interaction timescale for protons is inversely proportional to the density of matter in the post-shock region, where the particles are trapped by the magnetic field. Hydrodynamic simulations [19] indicate that the density in the shock region is larger than the unperturbed wind density, by a factor of $\delta \sim 1-100$. The p-p cooling time scale can therefore be written :

$$
t_{p p}=\frac{1}{\sigma_{p p} \delta n c}=\frac{4 \pi R^{2} m_{p} V_{w}}{\sigma_{p p} \delta \dot{M} c} \approx \frac{R_{10^{14} \mathrm{~cm}}^{2} V_{10^{3} \mathrm{~km} / \mathrm{s}}}{\delta_{10} \dot{M}_{10^{-4} \mathrm{M}_{\odot} / \mathrm{yr}}} \times 4 \cdot 10^{5} \mathrm{~s},
$$


where $V$ is the typical wind velocity and $\dot{M}$ is the mass loss rate of the primary star. Equating the acceleration time $t_{a c c}=\frac{R_{L}}{c}\left(\frac{c}{V}\right)^{2}$ respectively with $t_{I C}$ and $t_{p p}$ for electrons and protons $\left(R_{L}\right.$ is the Larmor radius) provides the maximum characteristic energy of the particle distribution. For electrons, a powerlaw spectrum is expected, with an exponential cutoff at

$$
\gamma_{\max , e}=\sqrt{\frac{3 \pi e c^{2}}{\sigma_{T} \beta^{2}}} \sqrt{\frac{B \cdot R^{2}}{L}} \frac{V}{c} \approx \sqrt{\frac{B_{1 \mathrm{G}} \cdot R_{10^{14} \mathrm{~cm}}^{2}}{L_{5 \cdot 10^{6} \mathrm{~L} \odot}}} V_{10^{3} \mathrm{~km} / \mathrm{s}} \times 3 \cdot 10^{4},
$$

where $B$ is the magnetic field in the shock region. $\gamma_{\max , e}$ should be fairly independent of the orbital position in the dipole approximation for a magnetic field varying as $R^{-2}$. The gamma-ray spectrum will therefore feature an exponential cutoff at the maximal energy [20]. A cutoff energy of $\sim 1$ $\mathrm{GeV}$ corresponds to a magnetic field of $\sim 50 \mathrm{G}$ at the stellar surface.

For protons, the maximum characteristic energy is limited by proton-proton interactions to

$$
\gamma_{\max , p}=\frac{4 \pi R^{2} e B}{\sigma_{p p} \delta \dot{M}}\left(\frac{V}{c}\right)^{3} \approx \frac{R_{10^{14} \mathrm{~cm}}^{2} B_{1 \mathrm{G}} V_{10^{3} \mathrm{~km} / \mathrm{s}}^{3}}{\delta_{10} \dot{M}_{10^{-4} \mathrm{M}_{\odot} / \mathrm{yr}}} \times 4 \cdot 10^{4} .
$$

The non-thermal spectral energy distribution of $\eta$ Carinae has been represented with a model consisting of two cutoff powerlaw distributions for the electrons and for the interacting protons. The model and the data are shown together in Fig. 1. The magnetic field and the electron energy distribution were adjusted to match the upper limit on the radio synchrotron emission, derived from the minimal thermal emission detected with ACTA [21], and to match the inverse Compton continuum determined by INTEGRAL and Fermi. The slope and cutoff energy of the interacting proton energy distribution were fixed to 2.25 and $10^{4}$, respectively, the same values as found for electrons. and its normalization was fitted to match the high-energy gamma-ray tail using $\pi^{0}$-decay[22]. The proton and ultraviolet photon energy densities in the shock region were fixed to the values expected for a representative distance of $R=10^{14} \mathrm{~cm}$.

\section{Energetic}

The wind momentum ratio of $\eta$ Carinae is $\eta=\left(\dot{M}_{2} V_{2}\right) /\left(\dot{M}_{1} V_{1}\right) \approx 0.2$ [23]. The half-opening angle of the shock region is therefore $\sim 1 \mathrm{rad}$, the fraction of the wind involved in the wind collision region is $\sim 10 \%$, and the mechanical energy available in that region is $\sim 200 \mathrm{~L}_{\odot}$. For a density of cold protons of $3 \cdot 10^{9} \mathrm{~cm}^{-3}$ in the shock region, the normalization of the $\pi^{0}$-decay spectrum requires a total interacting proton energy $E_{p} \sim 10^{40} \mathrm{erg}$. The energy injected in the shock to sustain the observed proton distribution is of the order of $E_{p} / t_{p p} \sim 10 \mathrm{~L}_{\odot}$. The gamma-ray spectrum of $\eta$ Carinae thus indicates that $\sim 5 \%$ of the shock mechanical energy (or less than $1 \%$ of the total wind mechanical luminosity) is transferred to accelerated protons downstream. This is in agreement with recent numerical simulations of relativistic collisionless shocks [24], if a significant fraction of the hadrons interacts and generates $\pi^{0}$.

Modeling the thermal X-ray emission depends on detailed hydrodynamical simulations[23] and could be affected by many phenomena [25]. Such simulations can explain an X-ray luminosity of $\approx 20 \mathrm{~L} \odot$ above a few $\mathrm{keV}$. Our analysis hence indicates that the fraction of the shock energy accelerating protons is similar to that emitting observable $\mathrm{X}$-rays in $\eta$ Carinae. 


\section{Conclusions}

We have detected a bright gamma-ray source at the position of $\eta$ Carinae. Its flux at a few $100 \mathrm{MeV}$ corresponds very well to the extrapolation of the hard X-ray spectrum of $\eta$ Carinae (as measured by INTEGRAL and Suzaku) towards higher energies. The spectral energy distribution, which corresponds to an average over almost half of the orbit of $\eta$ Carinae, features two spectral components.

The first one is a powerlaw extending from $\mathrm{keV}$ to $\mathrm{GeV}$ energies, with an exponential cutoff at $\sim 1 \mathrm{GeV}$. This component can be understood assuming inverse Compton scattering of stellar photons by electrons accelerated up to $\gamma \sim 10^{4}$ in the wind collision region. The observed cutoff energy implies a magnetic field of $\sim 50 \mathrm{G}$ at the stellar surface.

The second component, a hard gamma-ray tail, is detected above $20 \mathrm{GeV}$. This bright component could be explained by $\pi^{0}$-decay of accelerated hadrons interacting with the dense stellar wind in the shock region. The ratio between the fluxes of the $\pi^{0}$ and inverse Compton components is roughly as predicted by simulations [26,27]. Bremsstrahlung emission is expected at a much lower level and with a cutoff energy similar to that of the inverse Compton component.

The hard gamma-ray tail can only be understood if emitted close to the wind collision region. Indeed, the external shock between the Homunculus nebula and the interstellar medium occurs at a density and magnetic field strength that are much too small to explain the observed emission, even if a small fraction of the hard X-ray emission could come from inverse Compton scattering of infrared photons in the external shock [28, 17].

The energy transmitted to the accelerated particles ( $\sim 5 \%$ of the wind collision mechanical energy) is of the same order as that of the observed thermal X-ray emission, providing an important observational constraint for future numerical hydrodynamical models of the colliding wind region in $\eta$ Carinae.

\section{References}

[1] Hillas, A. M., ArXiv Astrophysics e-prints (2006).

[2] Strong, A. W. et al., ApJ 722 (2010) L58.

[3] Binns, W. R. et al., New A Rev.52 (2008) 427.

[4] Rauch, B. F. et al., ApJ 697 (2009) 2083.

[5] Abdo, A. A. et al., ApJ 710 (2010) L92.

[6] Abdo, A. A. et al., Science 327 (2010) 1103.

[7] Abdo, A. A. et al., ApJ 712 (2010) 459.

[8] De Becker, M., A\&A Rev. 14 (2007) 171.

[9] Benaglia, P. and Romero, G. E., A\&A 399 (2003) 1121.

[10] Axford, W. I., Annals of the New York Academy of Sciences 375 (1981) 297.

[11] Casse, M. and Paul, J. A., ApJ 258 (1982) 860.

[12] Smith, N., ApJ 644 (2006) 1151. 
[13] Davidson, K. and Humphreys, R. M., ARA\&A 35 (1997) 1.

[14] Hillier, D. J., Davidson, K., Ishibashi, K., and Gull, T., ApJ 553 (2001) 837.

[15] Viotti, R. F., Antonelli, L. A., Rossi, C., and Rebecchi, S., A\&A 420 (2004) 527.

[16] Leyder, J., Walter, R., and Rauw, G., A\&A 477 (2008) L29.

[17] Leyder, J., Walter, R., and Rauw, G., A\&A 524 (2010) A59+.

[18] Sekiguchi, A. et al., PASJ 61 (2009) 629.

[19] Pittard, J. M., MNRAS 396 (2009) 1743.

[20] Eichler, D. and Usov, V., ApJ 402 (1993) 271.

[21] Duncan, R. A. and White, S. M., MNRAS 338 (2003) 425.

[22] Kelner, S. R., Aharonian, F. A., and Bugayov, V. V., Phys. Rev. D74 (2006) 034018.

[23] Pittard, J. M. and Corcoran, M. F., A\&A 383 (2002) 636.

[24] Spitkovsky, A., ApJ 682 (2008) L5.

[25] Parkin, E. R., Pittard, J. M., Corcoran, M. F., Hamaguchi, K., and Stevens, I. R., MNRAS 394 (2009) 1758.

[26] Pittard, J. M. and Dougherty, S. M., MNRAS 372 (2006) 801.

[27] Reimer, A., Pohl, M., and Reimer, O., ApJ 644 (2006) 1118.

[28] Ohm, S., Hinton, J. A., and Domainko, W., ApJ 718 (2010) L161. 